\title{
Editorial: Earth-affecting Solar Transients
}

\author{
Jie Zhang ${ }^{1}$ (D) Xochitl Blanco-Cano $^{2}$ (D) \\ Nariaki Nitta $^{3}(D) \cdot$ Nandita Srivastava $^{4,5}$ (D) \\ Cristina H. Mandrini ${ }^{6,7}$
}

Published online: 9 May 2018

(C) Springer Science+Business Media B.V., part of Springer Nature 2018

This Topical Collection (TC) is devoted to the recent advancement in the study of Earthaffecting solar transients. Earth-affecting solar transients encompass a broad range of phenomena, including major solar flares, coronal mass ejections (CMEs), interplanetary CMEs (ICMEs), solar energetic particle (SEP) events, and corotating interaction regions (CIRs). In the past decade, nearly continuous observations of the Sun and the inner heliosphere

Earth-affecting Solar Transients

Guest Editors: Jie Zhang, Xochitl Blanco-Cano, Nariaki Nitta, and Nandita Srivastava

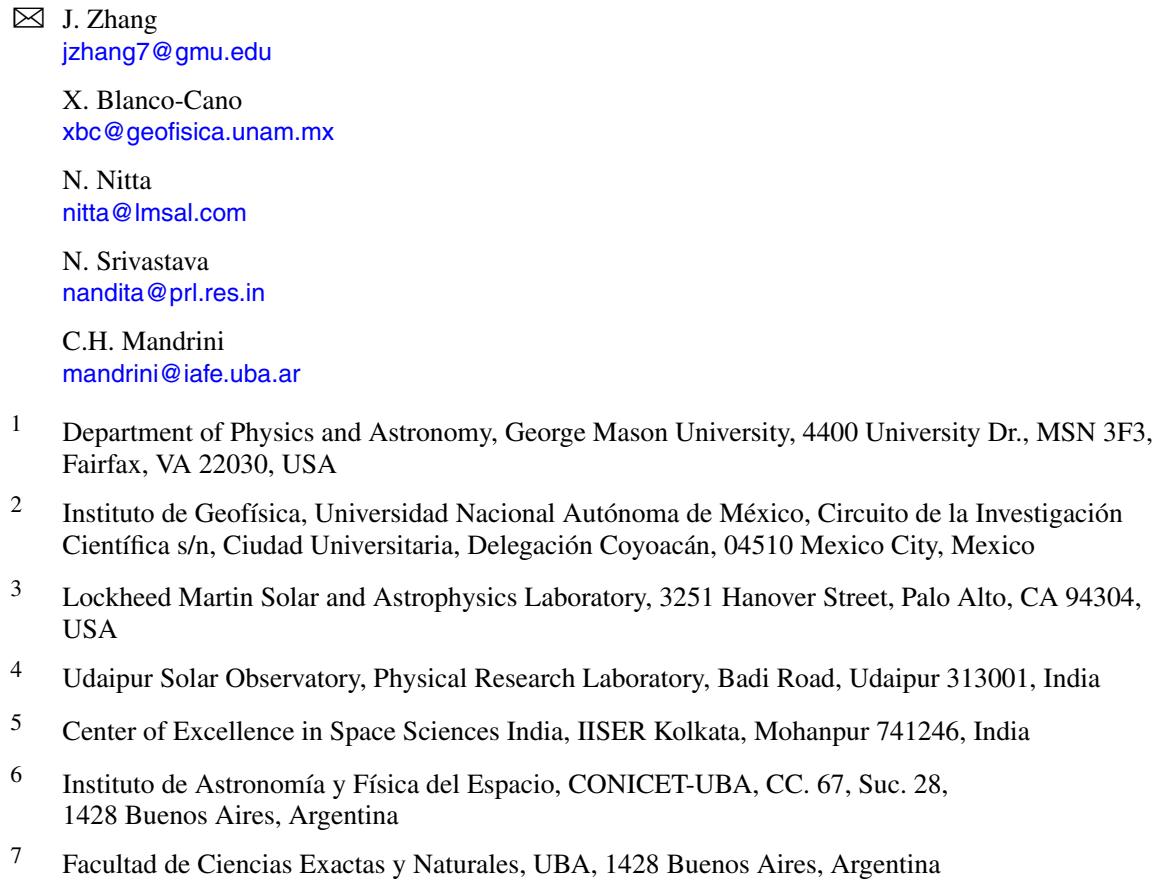


with an unprecedented wide spatial coverage from a fleet of spacecraft, including the Solar Terrestrial Relations Observatory Ahead/Behind (STEREO A/B), the Solar Dynamics Observatory (SDO), the Solar and Heliospheric Observatory (SOHO), the Mercury Surface, Space Environment, Geochemistry, and Ranging (MESSENGER) spacecraft, Venus Express (VEX), the Advance Composition Explorer (ACE), and Wind, in combination with a significant development and improvement of global magnetohydrodynamics (MHD) numerical simulations and theoretical analyses, have greatly improved our understanding of solar transients and the prediction of their potential impact on Earth. This TC includes articles that address, but are not limited to, the following questions: (1) How do various geoeffective phenomena originate on the Sun? (2) How do they propagate and evolve in the inner heliosphere? (3) How can we reconcile in situ and remote-sensing data on transients? (4) How can we predict the probability of arrival, time of arrival, and geoeffectiveness of these phenomena? (5) Which type of solar wind transients are geoeffective, and why?

This Topical Collection is based on the International Study of Earth-affecting Solar Transients (ISEST) project, initially launched in 2013 to bring together scientists from many countries to join efforts on studying solar transients. ISEST became one of the four research projects of the Variability of the Sun and Its Terrestrial Impact (VarSITI)) program, sponsored by the Scientific Committee on Solar-Terrestrial Physics (SCOSTEP) for the period of 2014-2018. The overarching goal of the ISEST project is to understand the origin, propagation, and evolution of solar transients through the space between the Sun and the Earth, and develop the prediction capability of space weather. Toward this goal, the ISEST project has organized three workshops in three different geographic locations across the globe: $17-$ 20 June 2013 in Hvar, Croatia, 26-30 October 2015 in Mexico City, Mexico, and 18-22 September 2017 in Jeju, South Korea. Several smaller but more focused workshops were also organized in convenient locations, e.g. as part of international meetings. The ISEST project maintains a standing website for hosting events catalogs, data, and presentations, and offers a forum for discussion at http://solar.gmu.edu/heliophysics/index.php/.

This Topical Collection contains 34 articles covering a broad range of scientific topics relevant to solar transients that can be separated into six large groups, which we summarize in the following six paragraphs.

In creating event catalogs, Hess and Zhang (2017) compiled a catalog of 70 Earthaffecting ICMEs in Solar Cycle 24 and tracked these events back to the sources of the eruptions in the low corona; the tracking was made possible possible through the complete Sun-to-Earth coverage of STEREO observations. These authors additionally made a statistical study of the properties of these events, including the source regions. Using more than 20 years of Wind observations, Nieves-Chinchilla et al. (2018) compiled a comprehensive database of ICMEs through three solar cycles and studied the asymmetry of the magnetic field strength profiles of these events. Ameri and Valtonen (2017) studied the occurrence and characteristics of geomagnetic storms associated with 66 disk-center full-halo CMEs from 1996 to 2015, of which 50\% were deduced to be the cause of 30 geomagnetic storms with Dst $\leq-50 \mathrm{nT}$. There are two review articles in this TC. Lugaz et al. (2017) presented a review of the different aspects associated with the interaction of successive CMEs in the corona and inner heliosphere, focusing on the initiation of series of CMEs, their interaction in the heliosphere, the particle acceleration associated with successive CMEs, and the effect of compound events on Earth's magnetosphere. Shen et al. (2017) reviewed the collision nature of two CMEs and pointed out that these collisions can have a different nature, i.e. inelastic, elastic, and super-elastic processes, depending on their initial kinematic characteristics.

In relation to the evolution of transients near the Sun, Kay et al. (2017) studied the deflection and rotation of seven CMEs, which originated from active region (AR) 11158 between 
13 and 16 February 2011, and found good agreement between observations and the Forecasting a CME's Altered Trajectory (ForeCAT) model. Nitta and Mulligan (2017) studied the origin of CMEs that were not accompanied by obvious low coronal signatures (LCSs), the so-called stealth CMEs, but were responsible for appreciable disturbances at $1 \mathrm{AU}$. These authors found that all these CMEs typically started slowly. Kim et al. (2017) investigated the relation of CME speed and magnetic helicity in CME source regions during the early phases of Solar Cycles 23 and 24. Ichimoto et al. (2017) presented a new solar imaging system for observing high-speed eruptions, the Solar Dynamics Doppler Imager (SDDI), whose great dynamic range in line-of-sight velocity measurements in $\mathrm{H} \alpha$ allows determining the motion of erupting filaments in 3D space.

To study the chain of events from the Sun to the Earth, Temmer et al. (2017) comprehensively examined the flare and CME event from 1 October 2011, covering the complete Sun-to-Earth chain of effects, including the evolution of the CME mass and the comparison of the magnetic flux inferred from remote-sensing and in situ observations. Using a large array of ground-based and satellite instruments, Piersanti et al. (2017) made a comprehensive analysis of the CME launched on 21 June 2015 and its specific effects on the magnetosphere, plasmasphere, and ionosphere. Rodkin et al. (2017) studied the kinematic and thermodynamic properties of the CMEs that occurred on 2-4 August 2011 and modeled their charge states to be consistent with in situ observations through estimating a probable heating rate of the CME plasma. Srivastava, Mishra, and Chakrabarty (2018) reported on the kinematics of two interacting CMEs observed on 13 and 14 June 2012 and found that the interaction of the two CMEs was inelastic in nature and led to the strongest sudden storm commencement $(\mathrm{SSC})(\approx 150 \mathrm{nT})$ in the current Solar Cycle 24. Ojeda-González et al. (2017) used six different methods, including minimum variance analysis and Grad-Shafranov (GS) reconstruction, to evaluate the properties of the complex ejecta in the time series of solar wind data obtained on 19-22 March 2001. They found that the event was composed of two magnetic clouds (MCs). Aslam and Badruddin (2017) studied the similarities and differences in the geoeffectiveness and galactic cosmic-ray (GCR) responses of selected ISEST campaign events. Watari (2018) identified and studied two geomagnetic storms associated with coronal holes within the 19 intense geomagnetic storms that occurred in Cycle 24. Bocchialini et al. (2018) selected 32 SSC events during 2002 as a starting point and performed a multidata analysis based on observations to associate them with their solar sources, identify their effects in the interplanetary medium, and analyzed the response of the terrestrial ionized and neutral environment.

In the context of magnetic properties of CMEs, Gopalswamy et al. (2017) reported a new method for computing the flare reconnection flux from post-eruption arcades and the underlying photospheric magnetic field. These authors found that the reconnection flux is correlated with the poloidal flux of the associated MC at 1 AU. Bothmer and Mrotzek (2018) made a comparison study of the 3D orientation of CMEs near the Sun and in situ and suggested that the kink in the near-Sun structure of the CMEs could explain the orientation differences. Sachdeva et al. (2017) analyzed the observed evolution of a set of 38 CMEs and found that the Lorentz forces acting on CMEs generally peak between 1.65 and $2.45 \mathrm{R}_{\odot}$. These forces become negligible in comparison to aerodynamic drag as early as 3.5 to $4 \mathrm{R}_{\odot}$ for fast CMEs and only from $12-50 \mathrm{R}_{\odot}$ for slow CMEs. Patsourakos and Georgoulis (2017) extended their earlier work on a helicity-based method to infer the CME magnetic field in both the Sun and the geospace through a generalization of the formalism to several possible flux rope configurations. These authors further discussed the implications for exoplanet habitability. Fitting several in situ CMEs with cylindrical and toroidal force-free flux rope models, Marubashi, Cho, and Ishibashi (2017) compared the orientation of these flux ropes with 
that of the magnetic polarity inversion lines (PILs) in their solar source regions. They argued that in most cases, the magnetic flux rope structure created in the corona is carried through interplanetary space and maintains its orientation. $\mathrm{Hu}$ (2017) developed an approach of the GS reconstruction for toroidal structures in space plasmas and made benchmark studies to demonstrate the performance of the GS solver. Based on 13 simple MC events with slow expansion speed and weak asymmetry, Al-Haddad et al. (2018) compared three reconstruction methods and suggested that attempts at reconciling in situ and remote-sensing views of CMEs must take into consideration the compatibility of different models with specific CME structures.

From in situ solar wind observations, Vršnak et al. (2017) examined CIRs during the declining phase of Solar Cycle 23 and found a generally weak correlation between solar wind plasma and magnetic field parameters and geomagnetic activity indices (the Dst, Ap, and AE indices and the Dst time derivative). Wu et al. (2017) examined a short-duration (35 min), but extremely dense (peak $94 \mathrm{~cm}^{-3}$ ), pulse in the solar wind and suggested that the high-density pulse might be the result of the compression of the heliospheric plasma sheet by an interplanetary shock crossing or an interaction between an interplanetary shock and a CIR. Applying a double superposed epoch analysis method to a large number of transients in the solar wind from 1976 to 2000, Yermolaev et al. (2017) argued that the observed differences between MC and non-MC ejecta arise because the satellite passes farther from the nose in non-MC than in MC ejecta. Yang et al. (2018) reported a study of the correlation between the solar wind magnetic field, $B$, and plasma parameters (density, $N$, temperature, $T$, and velocity, $V$ ) and found that while the magnetic field does not appear to be correlated with any individual plasma parameter, the field correlates well with the combined parameters $\sqrt{N V^{2}}$ and $\sqrt{N T}$. Augusto et al. (2017) studied a muon excess event at sea level on 28 August 2015 and attributed the increase to the acceleration of particles by shock waves at the front of the high-speed stream and the focusing effect of the heliospheric current sheet crossing. Using both observations and MHD simulations, Alves et al. (2017) evaluated the ability of two different solar wind transient events to generate ultra-low-frequency (ULF) waves in the equatorial region of the inner magnetopshere, which lead to a dropout in the relativistic electron fluxes in the outer radiation belt. Nina et al. (2018) studied the relationship between the solar X-ray radiation intensity and the D-region electron density in the Earth's ionosphere.

In the field of space weather prediction, Dumbović et al. (2017) presented a validation of the Coronal Mass Ejections and Solar Energetic Particles (COMESEP) space weather alert system and pointed out that the success rate of the system in its current form is unacceptably low for realistic operation. These authors further discussed the implications of a possible improvement of the alert system. Using a set of well-selected events, Webb and Nitta (2017) highlighted the problems in forecasting ICMEs and their geoeffectiveness. They identified likely source CMEs and found that the related solar surface activity ranged from uncertain or weak to X-class flares, while the geoeffects ranged from none to severe. Chertok, Grechnev, and Abunin (2017) extended their previously developed tool for SOHO data to SDO data. This tool allows for the early diagnostics of the geoeffectiveness of solar eruptions based on the estimate of the total unsigned line-of-sight photospheric magnetic flux in accompanying EUV arcades and dimmings.

In short, the articles in this Topical Collection have addressed a broad range of subjects relevant to Earth-affecting solar transients. We hope that readers enjoy this TC and find it valuable for their own work.

Acknowledgements The International Study of Earth-affecting Solar Transient (ISEST) project is cochaired by Jie Zhang, Manuela Temmer, and Nat Gopalswamy. We thank Bojan Vršnak for hosting the first 
ISEST workshop in Hvar, Croatia, in 2013, Alejandro Lara for hosting the workshop in Mexico City, Mexico, in 2015, and Kyungsuk Cho for hosting the workshop in Jeju, South Korea, in 2017.

\section{References}

Al-Haddad, N., Nieves-Chinchilla, T., Savani, N.P., Lugaz, N., Roussev, I.I.: 2018, Fitting and reconstruction of thirteen simple coronal mass ejections. Solar Phys. 293, 73. DOI.

Alves, L.R., Souza, V.M., Jauer, P.R., da Silva, L.A., Medeiros, C., Braga, C.R., Alves, M.V., Koga, D., Marchezi, J.P., de Mendonça, R.R.S., Dallaqua, R.S., Barbosa, M.V.G., Rockenbach, M., Dal Lago, A., Mendes, O., Vieira, L.E.A., Banik, M., Sibeck, D.G., Kanekal, S.G., Baker, D.N., Wygant, J.R., Kletzing, C.A.: 2017, The role of solar wind structures in the generation of ULF waves in the inner magnetosphere. Solar Phys. 292, 92. DOI. ADS.

Ameri, D., Valtonen, E.: 2017, Investigation of the geoeffectiveness of disk-centre full-halo coronal mass ejections. Solar Phys. 292, 79. DOI. ADS.

Aslam, O.P.M., Badruddin: 2017, Study of the geoeffectiveness and galactic cosmic-ray response of VarSITIISEST campaign events in Solar Cycle 24. Solar Phys. 292, 135. DOI. ADS.

Augusto, C.R.A., Navia, C.E., de Oliveira, M.N., Nepomuceno, A.A., Kopenkin, V., Sinzi, T.: 2017, Muon excess at sea level during the progress of a geomagnetic storm and high-speed stream impact near the time of Earth's heliospheric sheet crossing. Solar Phys. 292, 107. DOI. ADS.

Bocchialini, K., Grison, B., Menvielle, M., Chambodut, A., Cornilleau-Wehrlin, N., Fontaine, D., Marchaudon, A., Pick, M., Pitout, F., Schmieder, B., Regnier, S., Zouganelis, I.: 2018, Statistical analysis of solar events associated with storm sudden commencements over one year of solar maximum during Cycle 23: Propagation from the Sun to the Earth and effects. Solar Phys. 293, 75. DOI.

Bothmer, V., Mrotzek, N.: 2018, Comparison of CME and ICME structures derived from remote-sensing and in situ observations. Solar Phys. 292, 157. DOI. ADS.

Chertok, I.M., Grechnev, V.V., Abunin, A.A.: 2017, An early diagnostics of the geoeffectiveness of solar eruptions from photospheric magnetic flux observations: The transition from SOHO to SDO. Solar Phys. 292, 62. DOI. ADS.

Dumbović, M., Srivastava, N., Rao, Y.K., Vršnak, B., Devos, A., Rodriguez, L.: 2017, Validation of the CME geomagnetic forecast alerts under the COMESEP alert system. Solar Phys. 292, 96. DOI. ADS.

Gopalswamy, N., Yashiro, S., Akiyama, S., Xie, H.: 2017, Estimation of reconnection flux using post-eruption arcades and its relevance to magnetic clouds at 1 AU. Solar Phys. 292, 65. DOI. ADS.

Hess, P., Zhang, J.: 2017, A study of the Earth-affecting CMEs of Solar Cycle 24. Solar Phys. 292, 80. DOI. ADS.

Hu, Q.: 2017, The Grad-Shafranov reconstruction of toroidal magnetic flux ropes: Method development and benchmark studies. Solar Phys. 292, 116. DOI. ADS.

Ichimoto, K., Ishii, T.T., Otsuji, K., Kimura, G., Nakatani, Y., Kaneda, N., Nagata, S., UeNo, S., Hirose, K., Cabezas, D., Morita, S.: 2017, A new solar imaging system for observing high-speed eruptions: Solar Dynamics Doppler Imager (SDDI). Solar Phys. 292, 63. DOI. ADS.

Kay, C., Gopalswamy, N., Xie, H., Yashiro, S.: 2017, Deflection and rotation of CMEs from active region 11158. Solar Phys. 292, 78. DOI. ADS.

Kim, R.-S., Park, S.-H., Jang, S., Cho, K.-S., Lee, B.S.: 2017, Relation of CME speed and magnetic helicity in CME source regions on the Sun during the early phase of Solar Cycles 23 and 24. Solar Phys. 292, 66. DOI. ADS.

Lugaz, N., Temmer, M., Wang, Y., Farrugia, C.J.: 2017, The interaction of successive coronal mass ejections: A review. Solar Phys. 292, 64. DOI. ADS.

Marubashi, K., Cho, K.-S., Ishibashi, H.: 2017, Interplanetary magnetic flux ropes as agents connecting solar eruptions and geomagnetic activities. Solar Phys. 292, 189. DOI. ADS.

Nieves-Chinchilla, T., Vourlidas, A., Raymond, J.C., Linton, M.G., Al-haddad, N., Savani, N.P., Szabo, A., Hidalgo, M.A.: 2018, Understanding the internal magnetic field configurations of ICMEs using more than 20 years of wind observations. Solar Phys. 293, 25. DOI. ADS.

Nina, A., Cadez, V.M., Mitrovic Bajcetic, S.T.J., Popovic, L.C.: 2018, Analysis of the relationship between the solar X-ray radiation intensity and the D-region electron density using satellite and ground-based radio data. Solar Phys. 293, 64. DOI. ADS.

Nitta, N.V., Mulligan, T.: 2017, Earth-affecting coronal mass ejections without obvious low coronal signatures. Solar Phys. 292, 125. DOI. ADS.

Ojeda-González, A., Klausner, V., Mendes, O., Domingues, M.O., Prestes, A.: 2017, Characterization of the complex ejecta measured in situ on 19-22 March 2001 by six different methods. Solar Phys. 292, 160. DOI. ADS. 
Patsourakos, S., Georgoulis, M.K.: 2017, A helicity-based method to infer the CME magnetic field magnitude in Sun and geospace: Generalization and extension to Sun-like and M-dwarf stars and implications for exoplanet habitability. Solar Phys. 292, 89. DOI. ADS.

Piersanti, M., Alberti, T., Bemporad, A., Berrilli, F., Bruno, R., Capparelli, V., Carbone, V., Cesaroni, C., Consolini, G., Cristaldi, A., Del Corpo, A., Del Moro, D., Di Matteo, S., Ermolli, I., Fineschi, S., Giannattasio, F., Giorgi, F., Giovannelli, L., Guglielmino, S.L., Laurenza, M., Lepreti, F., Marcucci, M.F., Martucci, M., Mergè, M., Pezzopane, M., Pietropaolo, E., Romano, P., Sparvoli, R., Spogli, L., Stangalini, M., Vecchio, A., Vellante, M., Villante, U., Zuccarello, F., Heilig, B., Reda, J., Lichtenberger, J.: 2017, Comprehensive analysis of the geoeffective solar event of 21 June 2015: Effects on the magnetosphere, plasmasphere, and ionosphere systems. Solar Phys. 292, 169. DOI. ADS.

Rodkin, D., Goryaev, F., Pagano, P., Gibb, G., Slemzin, V., Shugay, Y., Veselovsky, I., Mackay, D.H.: 2017, Origin and ion charge state evolution of solar wind transients during 4-7 August 2011. Solar Phys. 292, 90. DOI. ADS.

Sachdeva, N., Subramanian, P., Vourlidas, A., Bothmer, V.: 2017, CME dynamics using STEREO and LASCO observations: The relative importance of Lorentz forces and solar wind drag. Solar Phys. 292, 118. DOI. ADS.

Shen, F., Wang, Y., Shen, C., Feng, X.: 2017, On the collision nature of two coronal mass ejections: A review. Solar Phys. 292, 104. DOI. ADS.

Srivastava, N., Mishra, W., Chakrabarty, D.: 2018, Interplanetary and geomagnetic consequences of interacting CMEs of $13-14$ June 2012. Solar Phys. 293, 5. DOI. ADS.

Temmer, M., Thalmann, J.K., Dissauer, K., Veronig, A.M., Tschernitz, J., Hinterreiter, J., Rodriguez, L.: 2017, On flare-CME characteristics from Sun to Earth combining remote-sensing image data with in situ measurements supported by modeling. Solar Phys. 292, 93. DOI. ADS.

Vršnak, B., Dumbović, M., Čalogović, J., Verbanac, G., Poljančić Beljan, I.: 2017, Geomagnetic effects of corotating interaction regions. Solar Phys. 292, 140. DOI. ADS.

Watari, S.: 2018, Intense geomagnetic storms associated with coronal holes under the weak solar-wind conditions of Cycle 24. Solar Phys. 293, 23. DOI. ADS.

Webb, D., Nitta, N.: 2017, Understanding problem forecasts of ISEST campaign flare-CME events. Solar Phys. 292, 142. DOI. ADS.

Wu, C.-C., Liou, K., Lepping, R.P., Vourlidas, A., Plunkett, S., Socker, D., Wu, S.T.: 2017, Observation of an extremely large-density heliospheric plasma sheet compressed by an interplanetary shock at 1 AU. Solar Phys. 292, 109. DOI. ADS.

Yang, Z., Shen, F., Zhang, J., Yang, Y., Feng, X., Richardson, I.G.: 2018, Correlation between the magnetic field and plasma parameters at 1 AU. Solar Phys. 293, 24. DOI. ADS.

Yermolaev, Y.I., Lodkina, I.G., Nikolaeva, N.S., Yermolaev, M.Y.: 2017, Dynamics of large-scale solar-wind streams obtained by the double superposed epoch analysis: 2. Comparisons of CIRs vs. sheaths and MCs vs. ejecta. Solar Phys. 292, 193. DOI. ADS. 